\title{
Secundarismo sifilítico en infección por VIH, con fenómeno de Prozona
}

\author{
Seeondary syphilis in 11 W infeetion, with prozone- \\ phenomenon
}

\author{
Gabriel Mosquera, Isabel Cuéllar, Margarita Tamayo, laura Charry • \\ BogotÁ, D.C. (Colombia)
}

\begin{abstract}
Resumen
La sífilis es una enfermedad infectocontagiosa, de transmisión sexual, con una expresión clínica muy variada, que tiene clara relación la evolución clínica, tiempo de infección e inmunidad del huésped. En los pacientes con infección por VIH, las etapas de la sífilis pueden tener formas evolutivas diferentes, habitualmente más severas. En pacientes con sífilis e infección por VIH se puede presentar el fenómeno de Prozona, que consiste en serología VDRL no reactiva, falsamente negativa, que debemos tener en cuenta en estos pacientes para hacer estudios adicionales que nos encaminen a diagnóstico y tratamiento acertado. (Acta Med Colomb 2014; 39: 00-00).
\end{abstract}

Palabras claves: sifilis, test FTA-ABS, VIH, SIDA.

\begin{abstract}
Syphilis is an infeetious disease of sextal transmission, with a varied elinieal expression, which is elearly related elinieal outeome, time of infeetion and host immunity. In patients with HIV infeetion, syphilis stages may have different volutionary forms, ustally more severe. In patients with syphilis and HW infeetion ean oeeur Prozonephenomenon, which is non-reaetive VDRL serology, false negative, which must be eonsidered in these patients for additional studies that will lead us to diagnosis and streess full treatment. (Aeta Med Colomb 2014; 39: 00-00).

Keyswords: syphitis, FTA-ABS Test, IHW, A円S.
\end{abstract}

Dr. Gabriel Mosquera Klinger: Especialista en Medicina Interna, Pontificia Universidad Javeriana; Dras. Isabel Cuéllar, Margarita Tamayo y Laura Charry: Servicio de Dermatología, Hospital Universitario San Ignacio. Bogotá, D.C. (Colombia).

Correspondencia Dr. Gabriel Mosquera Klinger.

E-mail: $x * x$

Recibido: 24/X/2012 Aceptado: 17/XII/2013

\section{Introducción}

La sífilis es una infección de transmisión sexual producida por el treponema pallidum, el diagnóstico en la mayoría de veces es clínico (1).

La coinfección de sífilis con virus de inmunodeficiencia humana (VIH) es frecuente. La evolución natural de la sífilis se puede ver notablemente afectada, anotando que en esta coinfección se pueden presentar el paso de una fase a la siguiente de forma rápida, además las manifestaciones clínicas pueden ser atípicas. Con relación a la sífilis secundaria hay múltiples casos de manifestaciones cutáneas atípicas y la afectación visceral puede ser de expresión variable como manifestaciones nefrológicas como síndrome nefrótico, gastrointestinales como hepatitis, gastroenteritis, osteomusculares como poliartritis, osteítis, pulmonares neumonitis, derrame pleural $(2,3)$.

El fenómeno de Prozona consiste en una prueba No treponémica, que resulta falsamente negativa, y se presenta por elevados títulos de anticuerpos en el contexto de sífilis secundaria, tiene una incidencia del 1-2\% (3), que se puede incrementar hasta $10 \%$ en pacientes con infección por VIH. Es de gran importancia que ante los hallazgos clínicos sugestivos de sífilis secundaria, en pacientes con sospecha o confirmados con infección por VIH, ante un resultado inicialmente negativo de serología VDRL o RPR, informemos en el laboratorio de microbiología sobre practicar estas mismas pruebas con diluciones especiales, para lograr detectar estos pacientes con resultados falsamente negativos. Ya que hay gran impacto en el diagnóstico y en el tratamiento específico.

\section{Presentación de caso}

Paciente de 23 años, sin antecedentes previos de enfermedad, homosexual, sin pareja estable. No usa métodos de protección. Última relación sexual un mes previo a cuadro clínico.

Consultó con cuadro de tres días de evolución de fiebre, escalofríos, malestar general, lesiones en piel descritas como 
pápulas eritematosas en cara, cuello, y genitales. Fue evaluado en múltiples oportunidades en servicios de urgencias por medicina general, que describían hallazgos compatibles con exantema, urticaria, recibió varios esquemas de tratamiento sin tener mejoría por lo que un mes después consulta a dermatología.

En la evaluación inicial por dermatología describen los siguientes hallazgos clínicos, en genitales (Figura 1), en cara (Figura 2), en miembros superiores, inferiores y tórax (Figura 3,4), con estos hallazgos clínicos las impresiones diagnósticas fueron sífilis secundaria, y se propuso descartar

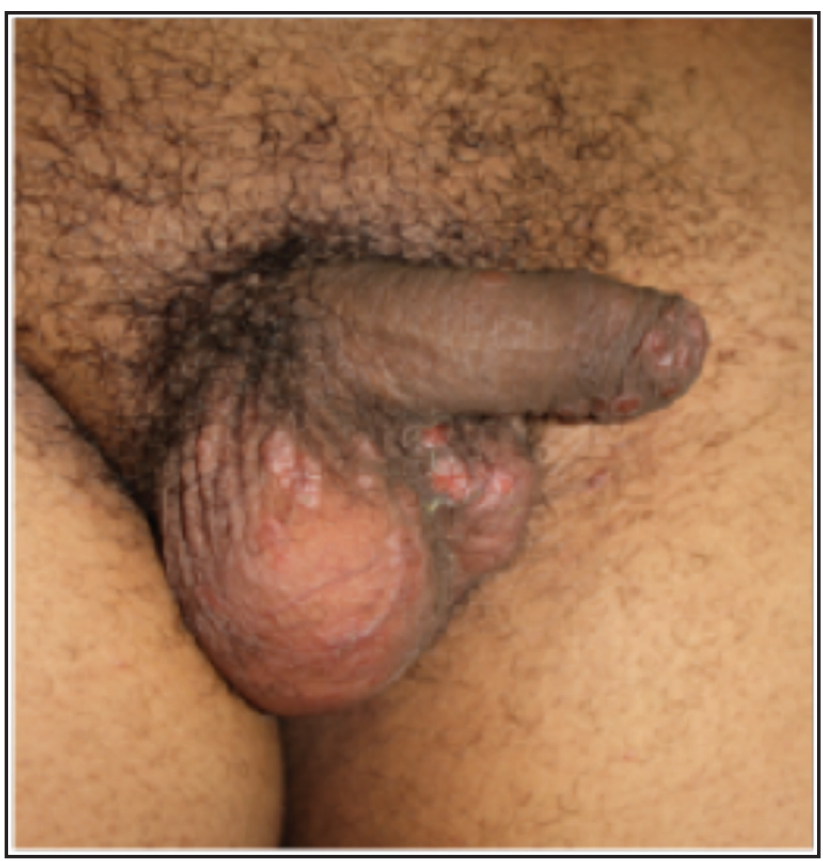

Figura 1. Pápulas eritemato violáceas infiltradas.

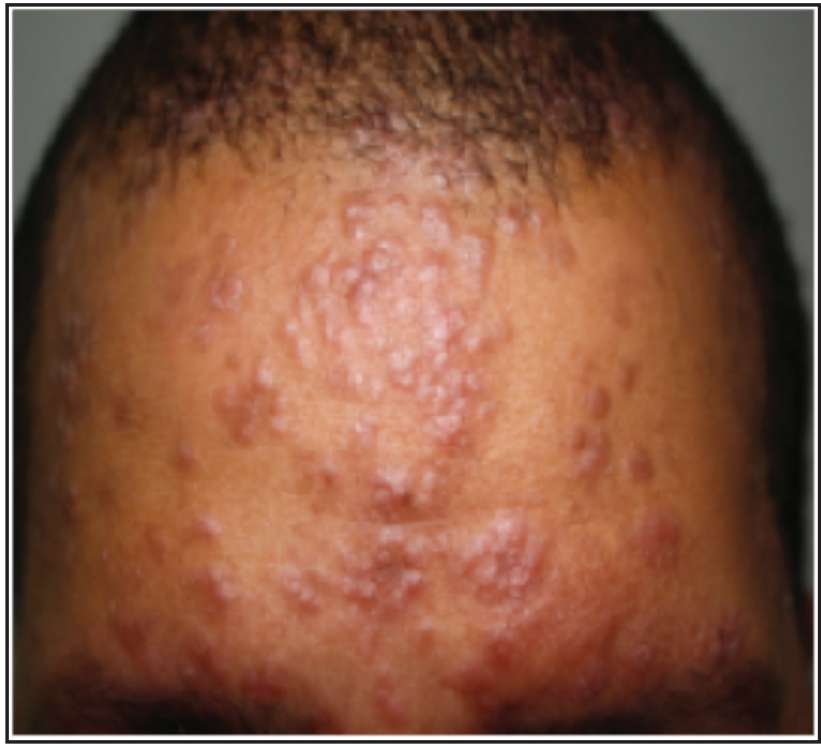

Figura 2. Pápulas y placas pardo eritemato violáceas infiltradas. coinfección por VIH, se le indicó VDRL que resultó No reactivo, ELISA VIH fue positivo, se confirmó el diagnóstico de infección por VIH con Western Blot. Se le tomó biopsia de piel en región escrotal, donde se documentaron hallazgos compatibles con sífilis secundaria (Figura 5).

Adicionalmente presentó cambios locales inflamatorios en maléolo externo izquierdo dados por dolor intenso, eritema, sin hallazgos de sinovitis, pero llamativo dolor local intenso a ese nivel. Se le indicó radiografía de tobillo izquierdo que fue normal, pero con una RMN de tobillo, donde no se documentó hallazgos de sinovitis, pero si se encontraron lesiones líticas en tercio distal de tibia, compatibles con osteítis de tibia distal.

Se le dio manejo médico con Penicilina Benzatínica 2.4 millones en dosis única IM durante dicha hospitalización.

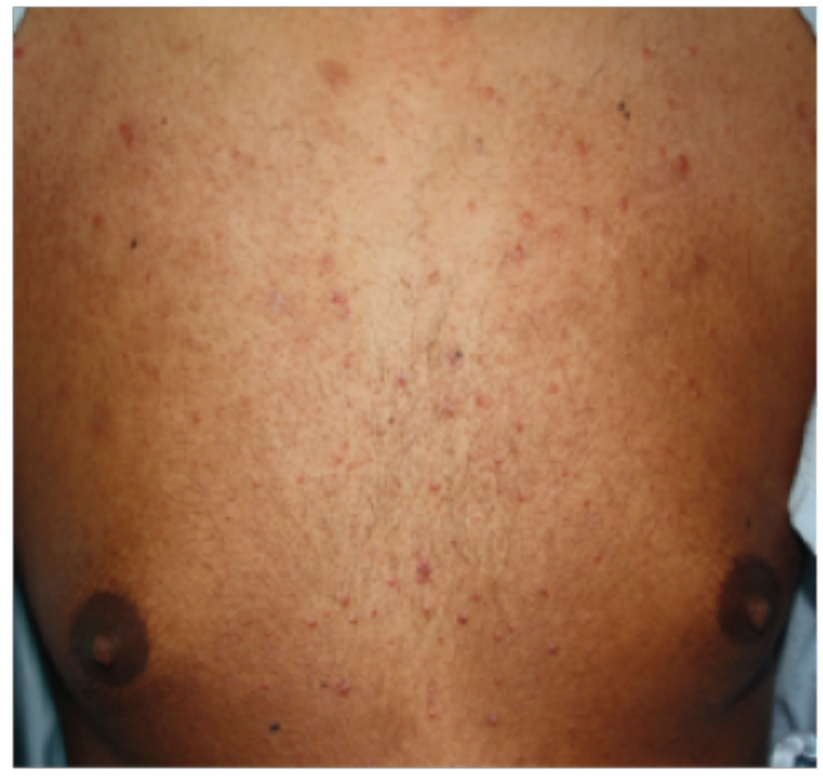

Figura 3. Múltiples pústulas de base eritematosa.

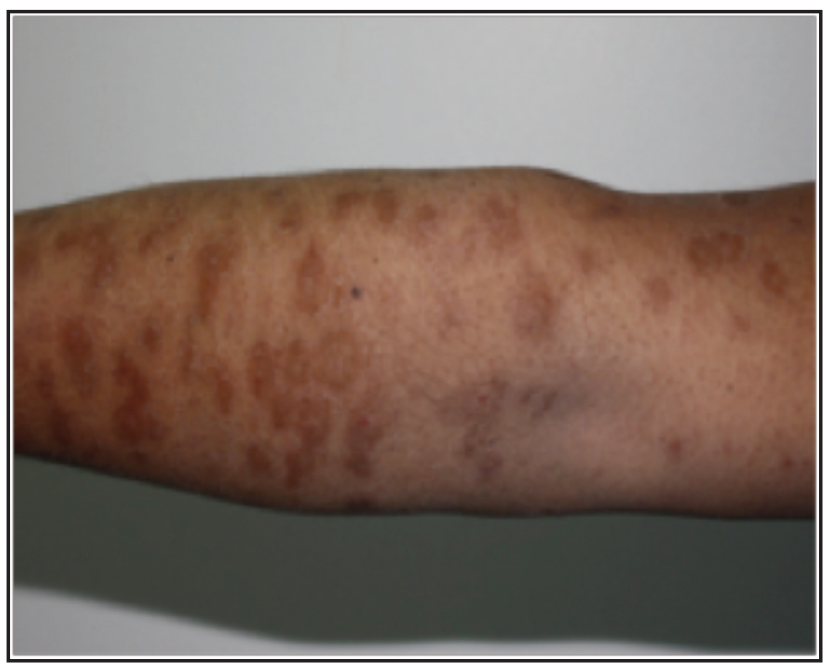

Figura 4. Placas pardo eritematosas redondeadas. 


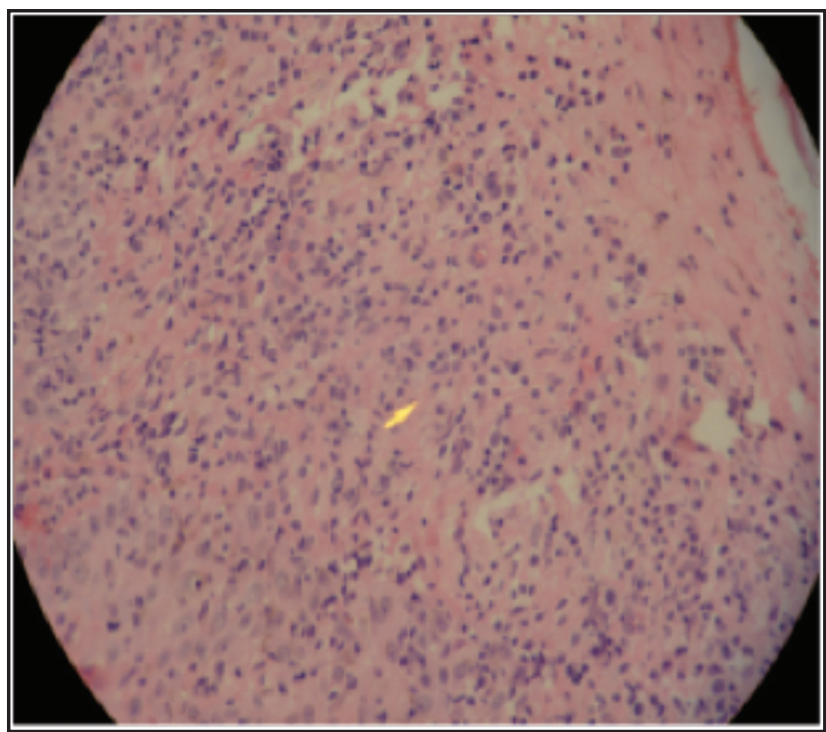

Figura 5. Biopsia de piel: Epidermis con hiperqueratosis e hiperplasia psoriasiforme y paraqueratosis. Dermis densamente infiltrada por plasmocitos con pobre formación de granulomas.

Con lo cual tuvo una mejoría clínica importante, mejoría de su estado general, mejoría en la apariencia de las lesiones en piel, mejoría de dolor y edema a nivel de maléolo externo.

Se le solicitó entonces FTA Abs que fue positivo, VDRL con diluciones por la sospecha del fenómeno de Prozona el cual fue reactivo, con lo que se realizó diagnóstico de sífilis secundaria en el contexto de infección reciente por VIH y fenómeno de prozona.

En los seguimientos por dermatología e infectología, se vio clara mejoría clínica, sin aparición de nuevos síntomas. Se dio de alta.

\section{Discusión}

La sífilis es llamada la gran imitadora, por las múltiples formas de presentación y manifestaciones clínicas. Afecta todos los sistemas y la severidad de los síntomas, así como la variabilidad tienen una clara relación con el estado inmunológico.

Se ha descrito una clara relación entre la coinfección por sífilis y por VIH, porque comparten el mismo medio de transmisión. En la sífilis secundaria y coinfección por VIH, se puede presentar fenómeno de Prozona por una alteración en la función de células B, con producción desproporcionada de Anticuerpos ante el treponema, presentándose así en estos pacientes un resultado falsamente negativo de las pruebas no treponémicas (RPR, VDRL) (3-5). En nuestro caso ante la fuerte sospecha de sífilis secundaria a pesar de la serología VDRL no reactiva, realizamos una nueva serología VDRL con diluciones con lo que se comprobó la falsa negatividad inicial de la prueba, como se ha descrito en otros reportes de casos $(3,5)$.
El fenómeno de Prozona se ha reportado en otras series, mayormente relacionado con coinfección por $\mathrm{VIH}(2,5)$, la recomendación que damos es hacer diluciones en todos los pacientes que tengan sospecha o confirmación de infección por VIH en los que tengan serología para sífilis negativa. Hasta el momento no ha habido publicaciones en este sentido en Colombia.

En cuanto a el tratamiento, la penicilina $\mathrm{G}$ administrada de forma parenteral es el medicamento de elección en todos los estadios de la sífilis (la preparación usada por ejemplo: benzatínica, cristalina), la dosis, duración del tratamiento, dependen de la etapa y manifestaciones clínicas de la enfermedad (1).

En nuestro caso le dimos tratamiento con una dosis de 2.4 millones de penicilina benzatínica, que está de acuerdo con las recomendaciones actuales dadas por la CDC 2010 (1). para el paciente con sífilis secundaria y VIH. Pero algunos autores han propuesto administrar penicilina benzatínica 2.4 millones cada semana por tres semanas hasta completar 7.2 millones, sin que haya una evidencia de su superioridad.

La recomendación actual de paciente con infección por $\mathrm{VIH}$, en sífilis latente, terciaria, o neurosífilis, es dar manejo con penicilina benzatínica 7.2 millones, dando 2.4 millones cada semana durante tres semanas, en estos casos si no hay respuesta serológica, o en LCR, entonces se debe administrar manejo con penicilina cristalina 4 millones cada 4 horas por 10-14 días.

En individuos inmunocompetentes con alergia a penicilinas se puede ofrecer tratamiento con doxiciclina $100 \mathrm{mg}$ 2 veces/día por dos semanas, tetraciclina 500mg cada 6 horas por 2 semanas, o ceftriaxone 1 gr/día por 10 días. En pacientes con Infección por VIH con historia de alergia a penicilinas con sífilis en cualquier estadio debe realizarse desensibilización, ya que las penicilinas son el tratamiento más efectivo en este grupo de pacientes (1). En todos los casos de sífilis debemos dar tratamiento a las parejas para evitar reinfección, así como búsqueda activa de otras enfermedades de transmisión sexual como la infección por VIH.

\section{Agradecimientos}

Agradecemos a el Dr. Luis Fernando Jaramillo, del servicio de patología del Hospital Universitario San Ignacio, que colaboró con el estudio histopatológico.

\section{Bibliografía}

1. Workowski K, Berman S. Centers for Disease Control and prevention Sexually Transmitted Diseases Treatment Guidelines. MMWR 2010; 59: 26-35.

2. Jurado RL, Campbell J, Martín PD. Prozone Phenomenon in secondary syphilis Has Its Time Arrived? Arch Intern Med 1993; 153: 2496-98.

3. Zaharopoulos $\mathbf{P}$, Wong J. Cytologic diagnosis of syphilitic pleuritis: a case report Diagn Cytopathol 1997; 16: 35-38.

4. Tramont, EC, Mandell GL, Bennett JE, Dolin R. Treponema pallidum (Syphilis). Principles and Practice of Infectious Diseases. Philadelphia, Churchill Livingstone, 2000; 5: 2484-2485.

5. Haslett P. The Prozone Phenomenon in Syphilis Associated with HIV Infection. Arch Int Med 1994; 154: 1643-1644. 